\title{
Annotations
}

\section{Brain death in children}

The recent brain death debate has been useful in at least two respects. Firstly, it appears to have prompted publication of the data which validate the underlying concept of brain death ${ }^{1}$-namely that with certain definable exceptions, absent brain stem reflexes are followed by asystole in all cases, generally within a matter of a few days, even if life support systems are continued. It is now apparent that the brain stem and the heart are dependent on each other for survival. That the brain stem is dependent on the heart has been evident for centuries. That the heart is dependent on the brain stem has rigorously been tested in the human only since the advent of mechanical ventilation. As the death of one automatically and in every case causes death of the other, we have had to get used to the idea that two forms of death exist. Secondly, the debate has emphasised the importance of the preconditions for the British criteria, ${ }^{2}$ the most important of which perhaps is that the diagnosis of brain death is only to be considered when there is 'no doubt that the patient's condition is due to irremediable structural brain damage,' and when the effects of drugs (including neuromuscular blocking agents), hypothermia, and severe metabolic derangements have been discounted. The debate may also have prompted us to re-examine our local facilities for detecting circulating neurodepressant drugs. It has reassured us that there is no need for haste in arriving at this diagnosis; indeed attempts to do so within 24 hours of the initial insult may be misleading.

The debate has sometimes been obscured by failure to understand that brain death is an operational term with clear prognostic implications. It is not a philosophical concept; it is pragmatic. In other words, the diagnosis of brain death when made by doctors always antedates death as generally understood hitherto-that is cessation of the heart beat. It does not demand that every cell in the brain be dead, fortunately perhaps because even with every modern technological resource brought to bear, it would not be possible to be absolutely certain that this was so (until dissolution set in). Electroencephalographical and arteriographical evidence is irrelevant.

An aspect of the debate which has not been sufficiently heard however, is the applicability of the British criteria to children. The question is particu- larly pertinent, for the case of a preterm infant who had brain stem failure after intraventricular haemorrhage and who subsequently survived intact is sometimes cited. ${ }^{3}$ However, all tests for brain stem failure were not carried out in that patient, and in any case intraventricular haemorrhage, generally arising from superficial fragile vessels in the germinal layer immediately subjacent to the ventricular surface, is not the same thing as irremediable structural brain damage. We know this to be a common event in preterm infants and one which is often clinically undetected. ${ }^{4}$ There is, to my knowledge, no case of survival in a child who met the British criteria in all the stated preconditions and exclusions. Since brain stem maturation is an early event in ontogeny, it is not to be expected that children would differ materially from adults in this respect. The question only becomes relevant therefore in the young infant who has suffered extensive head trauma or possibly extensive intracerebral haemorrhage secondarily to rupture of a congenital vascular malformation. Although this situation is rare, there is nevertheless a lack of evidence to allow a diagnosis of brain death to be made with complete confidence.

The situation more commonly seen in children is that of absent brain stem reflexes in the setting of complex multisystem disease which may have been punctuated by severe metabolic derangement and episodes of hypoxia. In such cases it may not be possible to be certain about mechanisms of brain damage. Here, as perhaps in the case of the smallest infants, there are good reasons for delay. One reason is the uncertainty about diagnosis. It is possible, for example, to envisage a brain stem encephalitis causing absent brain stem reflexes and depression of consciousness which is potentially reversible. Irreversible damage will become apparent in the course of the next few days because the heart will stop despite continuing support. While the issue awaits resolution the family will become aware that the child they knew no longer exists. It may be argued that delay is also useful for demonstration of an electroencephalogram which is isoelectric or nearly so, or a computerised tomography scan which shows widespread damage. While these results may be helpful in determining the likelihood of subsequent severe 
handicap, they add nothing to the clinical signs as far as the diagnosis of brain death is concerned. There is, in any case, nothing so convincing for the family as a period of time spent with an unaware and unresponsive, but ventilated child, to enable them to accept this fairly unfamiliar alternative form of death. The realisation that death has already occurred then makes stopping the ventilation seem a natural step in an ongoing process rather than a terminating event. The intervening period is also useful for the staff caring for the child to accept the child's death and thus allow them to support more effectively the grieving family.

Needless to say, the decision about whether and when to stop ventilation should never be influenced by organ transplant considerations. Furthermore, when mechanisms of brain damage are in doubt, nobody, child or adult, should be used as an organ donor until the heart has stopped beating despite continuing full life support.

This policy of delay is, of course, already widely advocated and practised, ${ }^{5}$ and does not generate vegetative survivors. While it can be argued that this may have been prevented by stopping ventilation in some, many have died despite continuing ventilation. Thus, 32 of the 198 brain-dead patients in the Glasgow series were children aged less than 15 years. Ventilation was continued on 13 of these despite brain death. All developed asystole (B Jennett, 1981, personal communication). It would be reassuring, notwithstanding the total lack of evidence to the contrary, to have this course of events adequately documented in all age groups.

Discontinuation of life support facilities in paediatric intensive care units (not excluding neonatal intensive care units) is frequently prompted less by consideration of brain death criteria than by the overwhelming probability of at least one potentially brain damaging event leading, in the unlikely event of survival, to severe handicap. Although again clearly this is never a decision to be made in haste, it is a quite separate and even more complex question to considerations of brain death. Here, ancillary investigations provide valuable confirmatory evidence and should be sought. All that needs to be said is that when a decision to stop ventilation has been made, there should be no doubt in anybody's mind, doctor, hurse, or family, of the reasons for so doing.

\section{References}

1 Jennett B, Gleave J, Wilson P. Brain death in three neurosurgical units. Br Med J 1981 ; 282 : 533-9.

2 Royal Colleges and Faculties of the United Kingdom. Diagnosis of brain death. Lancet 1976; ii: 1069-70.

3 Pasternak J F, Volpe J J. Full recovery from prolonged brain stem failure following intraventricular hemorrhage. J Pediatr 1979; 95 : 1046-9.

4 Papile L-A, Burstein J, Burstein R, Koffler H. Incidence and evolution of subependymal and intraventricular hemorrhage. A study of infants with birth weights less than 1500 g. J Pediatr 1978; 92: 529-34.

5 Freeman J M, Rogers M C. On death, dying, and decisions. Pediatrics 1980; 66: 637-8.

R O RoBINSON Newcomen Centre, Guy's Hospital, St Thomas Street, London SE1 9RT 